\title{
3D Structure of STAM1 UIM-ubiquitin Complex Using RosettaDock
}

\author{
Jongsoo Lim², Jong-Jae $\mathrm{Yi}^{1}$, Hee-Chul Ahn ${ }^{2}$, Jin-Kyu Rhee ${ }^{3}$, and Woo Sung Son ${ }^{1 *}$ \\ ${ }^{1}$ College of Pharmacy, CHA University, 198-1, Donggyo-dong, Pocheon-si, Gyeonggi-do 487-801, Republic of \\ Korea \\ ${ }^{2}$ College of Pharmacy, Dongguk University-Seoul, Goyang, Gyeonggi 410-820, Republic of Korea \\ ${ }^{3}$ Department of Chemistry and The Skaggs Institute for Chemical Biology, The Scripps Research Institute, \\ 10550 North Torrey Pines Road, La Jolla, CA 92037 \\ (Received May 5, 2011; accepted May 27, 2011)
}

\begin{abstract}
D structures of STAM1 UIM-ubiquitin complex were presented to predict and analyze the interaction between UIM and ubiquitin. To generate the protein-peptide complex structure, the RosettaDock method was used with and without NMR restraints. High resolution complex structure was acquired successfully and evaluated electrostatic interaction in the protein-peptide binding with several charged residues at the binding site. From docking results, the Rosettadock method could be useful to acquire essential information of protein-protein or protein-peptide interaction with minimal biological evidences.

Keywords : RosettaDock; Protein-Protein Complex; Ubiquitin; STAM1; ESCRT; lysosomal degradation; UIM; VHS domain
\end{abstract}

\section{INTRODUCTION}

One of the important post-translational modifications is ubiquitination of proteins and this modification regulates a variety of cellular processes ${ }^{1}$. During ubiquitin-mediated selective trafficking of membrane proteins, ubiquitinated membrane proteins should be delivered inside of the

\footnotetext{
* To whom correspondence should be addressed. E-mail : wson@cha.ac.kr
} 
cell via multivesicular bodies (MVBs) by endocytosis, and finally to lysosomes. And the endosomal sorting complex required for transport (ESCRT) complexes sort the ubiqutinated membrane proteins for lysosomal degradation ${ }^{2}$. Interestingly, the ESCRT-0 complex contains multiple ubiquitin binding domains, a Vps27/ Hrs/Stam (VHS) domain and a ubiquitin binding motif (UIM) for signal transducing adaptor molecule (STAM), and a VHS domain and a double-sided ubiquitin interacting motif (DUIM) for Hrs, respectively ${ }^{3}$.

Via those multiple ubiquitin binding domains, ESCRT-0 recognizes ubiquitinated cargo proteins, in which the VHS domain of STAM has higher binding affinity to K63-linked diubiquitin than to K48-linked diubuquitin ${ }^{3}$. To investigate the binding preference of ESCRT-0 to K63-linked di-, or poly-ubiquitin, it is essential to understand both individual VHS-ubiquitin and UIM-ubiquitin interactions. Previously, it has been determined a novel ubiquitin binding site on STAM1 VHS and suggested the model of VHS-ubiquitin interaction by using NMR spectroscopy ${ }^{4}$. In this work, the 3D structure of STAM1 UIM-ubiquitin complex is presented using RosettaDock method to predict and evaluate the interaction between the UIM motif and ubiquitin.

\section{EXPERIMENTAL}

\section{Peptide synthesis and protein preparation}

All STAM1 UIM peptide and ubiquitin protein were prepared as described previously ${ }^{5}$. 


\section{NMR experiments and structure calculation}

All NMR data were acquired at $298 \mathrm{~K}$ on Varian VNMRS $900 \mathrm{MHz}$ spectrometer with z-axis gradient equipment and STAM1 UIM peptide structures were calculated and validated as described previously $^{5}$.

\section{Protein-peptide docking}

STAM1 UIM-ubiquitin complex structures were generated using the RosettaDock method implemented with the Monte Carlo minimization ${ }^{6}$. Initial complex model based on NMR chemical shift perturbation analysis and structural alignment ${ }^{7,8}$ with VSP27 UIM-ubiquitin structure ${ }^{9}$ (PDB ID: 1Q0W) was used for starting protein-protein docking the surrounding free energy landscape by RosettaDock protocol. At first, the position of the UIM peptide was perturbed by random translations and rotations with the adjustment of distance between the peptide and ubiquitin to create contacts. Then, Monte Carlo energy minimization was used for optimizing the complex orientation. After lowresolution search, the explicit side chains were added to the backbones, and all-atom were optimized from a backbone-dependent rotamer library including the side chain conformations in the unbound proteins. The 100 best energy-scoring structures among 100,000 decoys from high-resolution docking simulation were clustered on the basis of pair-wise rmsd using a hierarchical clustering algorithm with van der Waals interactions, an implicit solvation model, and an orientation-dependent hydrogen 
bonding potential. Decoys within 0.3 A clustering threshold were selected as a final protein-peptide complex structure set. And if necessary, the high-resolution search was performed repeatedly with different initial structures. To validate final selected structures, FunHunt server was used ${ }^{10}$. Binding site of complex structure was analyzed using PIC analysis server ${ }^{11}$ and Protor $\mathrm{P}^{12}$. Protein-protein complex structures were analyzed and displayed with the software $\mathrm{MolMol}^{13}$ and Chimera ${ }^{7}$.
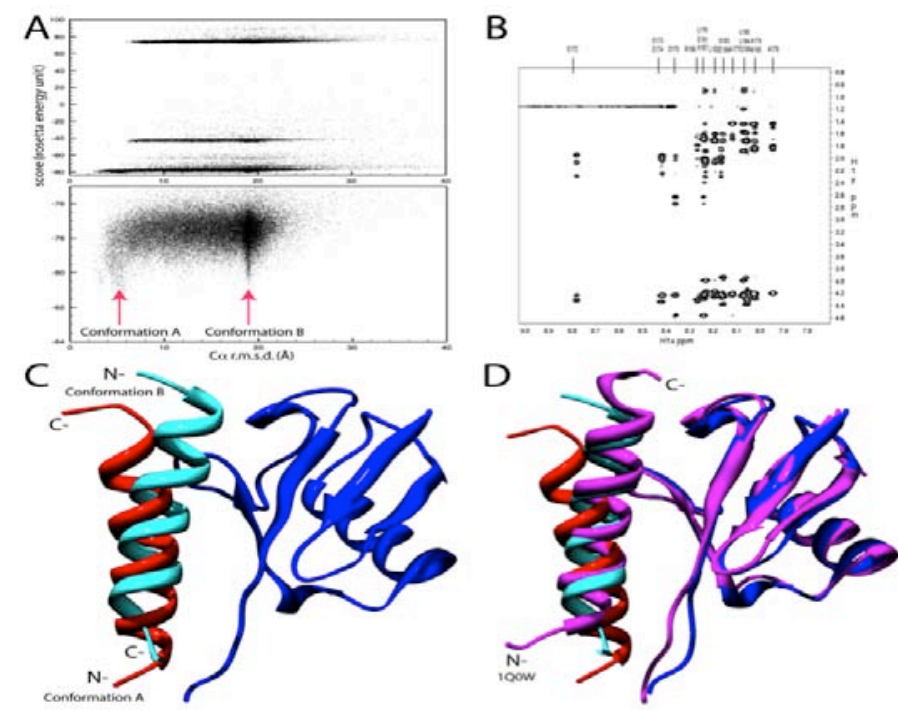

Figure. 1. STAM1 UIM-ubiquitin complex structure from random initial position using Rosetta-dock method. A: plot of $\mathrm{C} \alpha$ rmsd and Rosetta score. Two funnels were indicated with red arrows corresponding conformation A and conformation B. B: 2D NOESY spectrum of STAM1 UIM peptide. C: Ribbon presentation of STAM1 UIM-ubiquitin complex structure (blue: ubiquitin, red: conformation A, cyan: conformation B). D: Structural alignment with other UIM-ubiquitin complex structure (pdb ID: 1Q0W, colored by magenta). N-, C- means N-terminus and C-terminus regions of peptide, respectively. 


\section{RESULTS}

From protein-peptide docking simulation, 100,000 complex structures were generated with 2 funnels that could be close to native structure potentially. Each two conformation from funnels are different rmsd values to initial starting structure, $5 \AA$ (Conformation A) and $18 \AA$ (Conformation B) (Figure 1A). Two conformations have similar spatial position but opposite direction (Figure 1C). After complex structure validation with FunHunt method, the conformation A has the funnel in the perturbation with conformation B therefore, it was concluded that the conformation A is more reasonable structure that conformation $\mathrm{B}$. This was confirmed with the structural alignment with vps27 UIM-ubiquitin complex elucidated previously (Figure 1D) ${ }^{9}$.
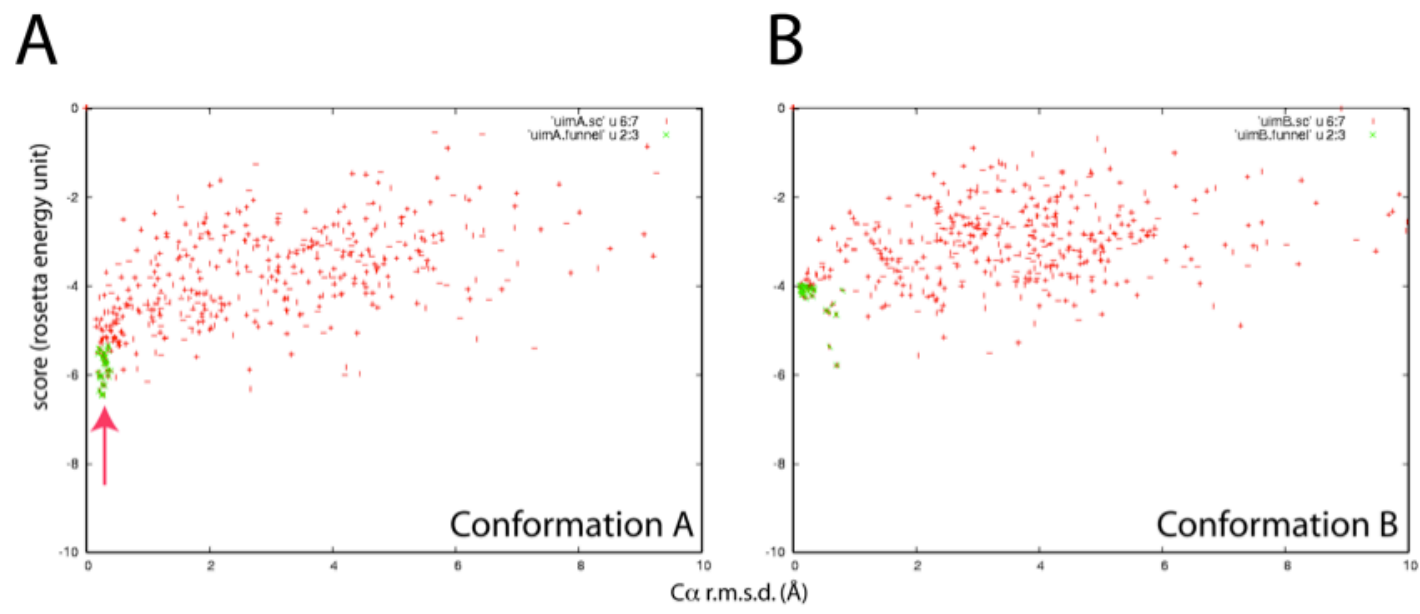

Figure. 2. Structure selection using FunHunt method. The weight vector used by FunHunt to discriminate between true (A) and false (B) funnels can be used to define characteristic features of true funnels. A sharp funnel toward 0 interface rmsd in A, can also indicate conformation A of two is the correct near native conformation. 


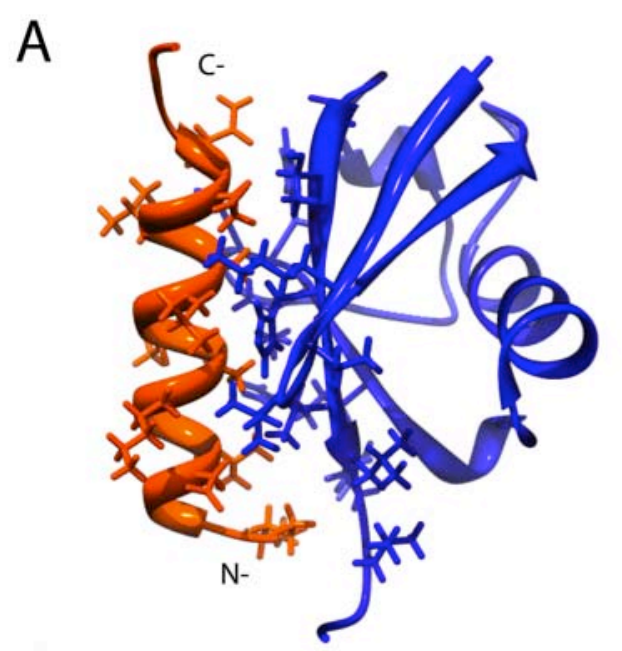

B

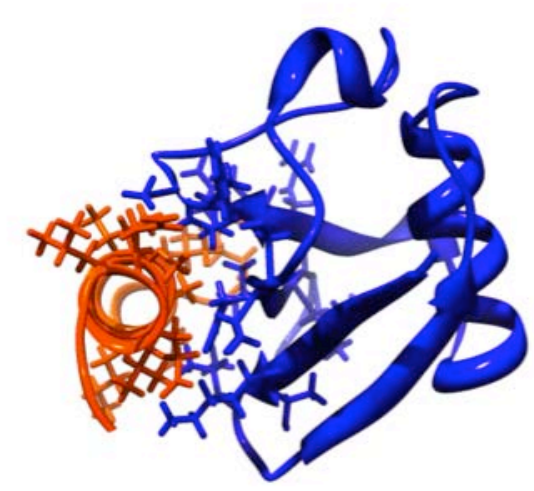

C

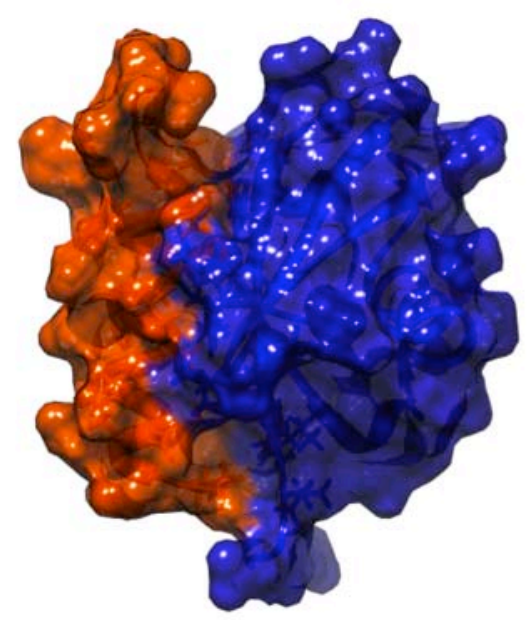

D

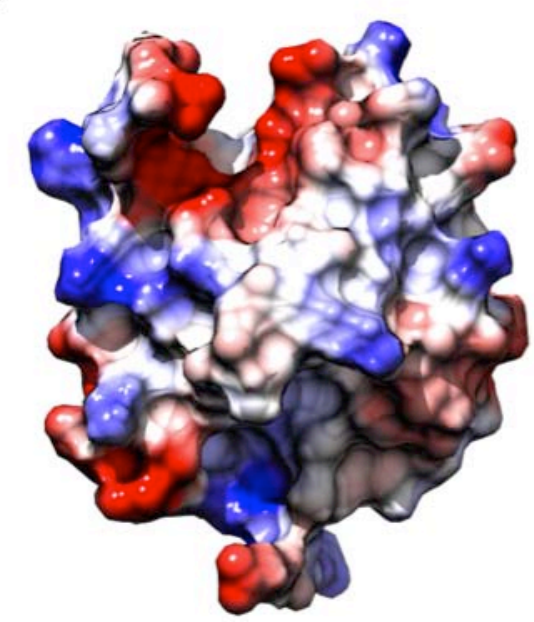

Figure. 3. Final STAM1 UIM-ubiquitin complex structures. Protein-peptide complex structures were displayed with ribbon presentation in side view (A) and top view (B). STAM1 UIM peptide was colored by orange red and ubiquitin protein was colored by blue, respectively and sidechain residues at the binding site were displayed with line presentation. Also, surface presentation (C) and electrostatic charge distribution (D, red for negative charge and blue for positive charge, respectively) of protein-peptide were displayed. 
86 Docking Structures of STAM1 UIM-ubiquitin

To get proper binding site information, generated protein-peptide complex structures were analyzed using PIC $^{11}$ and ProtorP $\mathrm{P}^{12}$ methods. There were 16 and 11 interface residues in the binding site of STAM1 UIM and ubiquitin including 3 hydrogen bonds, Glu186-Lys6, Ser183-Gly47, and Lys171-Leu73 (STAM1 UIM-ubiquitin pairs, respectively) (Figure. 3A, 3B). And major interface accessible surface area (ASA) residues were calculated as Lys171, Leu176, Ala179, Ile180 Leu182, Ser183, Glu186, and Gln187 in STAM1 UIM and Lys6, Leu8, Ala46, Thr66, and His68 in ubiquitin with $10 \%$ interface ASA criteria. Total interface ACA was $524.52 \AA$ and gap volume was $2315.25 \AA^{3}$ with 7 salt bridges using Protorp method. The electrostatic interaction is important to explain STAM1 UIM-ubiquitin binding except hydrophobic interaction. At the binding interface, STAM1 UIM and ubiquitin have several charged residues including Lys171, Asp175, Lys178, and Glu186 for STAM1 UIM and Lys1, Arg42, His68, and Arg72 for ubiquitin, respectively.

\section{DISCUSSION}

Protein-protein and protein-peptide docking is very useful to elucidation of protein structurefunction relationship and protein mechanism but is not easy to get full 3D complex structure using conventional structure determination method including X-ray crystallography and NMR due to limited structural information. In this paper, RosettaDock method was used to elucidating STAM1 UIM-ubiquitin complex structure with NMR restraints. Because STAM1 UIM peptide is short and 
very close to binding interface of ubiquitin, the incorporation of chemical shift perturbation (CSP) had little effect on protein-peptide docking. And 2 funnels with opposite direction of STAM1 UIM peptide was also originated from the shortness of peptide. Therefore, the wrong conformation structure (conformation B at Figure. 1A) could be eliminated with longer STAM1 UIM motif or full size protein.

Usually, the charged residues in the binding interfaces are important except hydrophobic interaction ${ }^{14}$. In the case of STAM1 UIM-ubiquitin binding, the several charged residues could play a key role in protein-peptide binding. From the result of protein-peptide docking, it could be suggested several positive charged residues and negative charged residues at the STAM1 UIM-ubiquitin binding site and the electrostatic interaction of these residues in STAM1 UIM-ubiquitin complex was confirmed with NMR and ITC experiments as published ${ }^{5}$. Therefore, in STAM1 UIM-ubiquitin binding study, the protein-peptide docking using RosettaDock method is very useful to estimate binding residues of each binding partner and essential to analyze the binding interaction. Considered results in this paper, the RosettaDock method could be applicable to protein-peptide and proteinprotein docking study with or without variant NMR restraints. 
88 Docking Structures of STAM1 UIM-ubiquitin

\section{Acknowledgements}

This research was supported by the Industry Academic Cooperation Foundation funded by the CHA University [CHAIACF-2011]. H.-C.Ahn was supported by a grant of the Korea Healthcare Technology R\&D Project, Ministry of Health and Welfare, Republic of Korea (A092006).

\section{REFERENCES}

1. V. Kirkin and I. Dikic, Curr Opin Cell Biol. 19, 199. (2007).

2. C. Raiborg and H. Stenmark, Nature 458, 445. (2009).

3. X. Ren and J. H. Hurley, EMBO J. 29, 1045. (2010).

4. A. Lange, D. Hoeller, H. Wienk, O. Marcillat, J. M. Lancelin, and O. Walker, Biochemistry 50, 48. (2011).

5. Y. H. Hong, H. C. Ahn, J. Lim, H. M. Kim, H. Y. Ji, S. Lee, J. H. Kim, E. Y. Park, H. K. Song, and B. J. Lee, FEBS Lett. 583, 287. (2009).

6. J. Lim, W. S. Son, J. K. Park, E. E. Kim, B. J. Lee, and H. C. Ahn, Biochemical and biophysical research communications 205, 24. (2011).

7. J. Gray, S. Moughon, C. Wang, O. Schueler-Furman, B. Kuhlman, C. Rohl, and D. Baker, J Mol Biol. 331, 281. (2003).

8. E. F. Pettersen, T. D. Goddard, C. C. Huang, G. S. Couch, D. M. Greenblatt, E. C. Meng, and T. E. Ferrin, J Comput Chem. 25, 1605. (2004).

9. M. S. Johnson, M. J. Sutcliffe, and T. L. Blundell, J Mol Evol. 30, 43. (1990).

10. E. Krissinel and K. Henrick, Acta Crystallogr D Biol Crystallogr. 60, 2256. (2004).

11. E. C. Meng, E. F. Pettersen, G. S. Couch, C. C. Huang, and T. E. Ferrin, BMC Bioinformatics 7, 339. (2006).

12. K. A. Swanson, R. S. Kang, S. D. Stamenova, L. Hicke, and I. Radhakrishnan, EMBO J. 22, 4597. (2003).

13. N. London and O. Schueler-Furman, Proteins 69, 809. (2007) 
14. N. London and O. Schueler-Furman, Structure 16, 269. (2008).

15. K. G. Tina, R. Bhadra, and N. Srinivasan, Nucleic Acids Res. 35, W473. (2007).

16. C. Reynolds, D. Damerell, and S. Jones, Bioinformatics 25, 413. (2009).

17. R. Koradi, M. Billeter, and K. Wüthrich, J Mol Graph. 14, 51. (1996).

18. E. Leonardi, S. Andreazza, S. Vanin, G. Busolin, C. Nobile, and S. C. E. Tosatto, PLoS ONE 6, e18142. (2011)

19. B. Busby, T. Oashi, C. D. Willis, M. A. Ackermann, A. Kontrogianni-Konstantopoulos, A. D. Mackerell, and R. J. Bloch, J Mol Biol. 408, 321. (2011). 